\title{
DUALITY THEOREMS AND AN OPTIMALITY CONDITION FOR NON-DIFFERENTIABLE CONVEX PROGRAMMING
}

\author{
P. KANNIAPPAN and SUNDARAM M. A. SASTRY
}

(Received 22 August 1980; revised 23 March 1981)

Communicated by $\mathrm{H}$. Lausch

\begin{abstract}
Necessary and sufficient optimality conditions of Kuhn-Tucker type for a convex programming problem with subdifferentiable operator constraints have been obtained. A duality theorem of Wolfe's type has been derived. Assuming that the objective function is strictly convex, a converse duality theorem is obtained. The results are then applied to a programming problem in which the objective function is the sum of a positively homogeneous, lower-semi-continuous, convex function and a continuous convex function.
\end{abstract}

1980 Mathematics subject classification (Amer. Math. Soc.): 90 C 25, 90 C 30, 90 C 48.

\section{Introduction}

In this paper, we study the following pair of problems:

Problem (P). Minimize $f(x)$ subject to

$$
G(x) \leqslant 0 \text { and } x \in A .
$$

Problem (D). Maximize $f(x)+\left\langle z^{*}, G(x)\right\rangle$ subject to

$$
\begin{aligned}
& z^{*} \geqslant 0, \quad x \in A \quad \text { and } \\
& 0 \in \partial f(x)+z^{*} \circ \partial G(x)+N(x / A) .
\end{aligned}
$$

The first author is on leave from Gandhigram Rural Institute (Deemed University), Gandhigram624302, India and his research is supported by U.G.C. of India (V plan).

This paper was presented at the Annual Conference of the Indian Mathematical Society, held in December 1980, at Bangalore, India.

c Copyright Australian Mathematical Society 1982 
Here $f$ is a continuous convex functional defined on a locally convex space $X$ and $G$ is a continuous convex operator, which is regularly subdifferentiable on $A$, a convex subset of $X$, defined on $X$ into another locally convex space $Z$ having a closed convex cone defining a partial ordering in $Z$. $N(x / A)$ denotes the normal cone to $A$ at $x$ defined by

$$
N(x / A)=\left\{w^{*} \in X^{\prime}:\left\langle w^{*}, y-x\right\rangle \leqslant 0 \text { for all } y \in A\right\},
$$

where $X^{\prime}$ is the dual space of $X$.

$N(x / A)$ is the subdifferential of the indicator function of the set $A$ at $x, \delta(x / A)$ defined by

$$
\delta(x / A)= \begin{cases}0 & \text { if } x \in A, \\ \infty & \text { if } x \notin A .\end{cases}
$$

If $X$ and $Z$ are finite dimensional, $f$ and $G$ are differentiable and $A=X$, then this is the problem studied by Wolfe and he has proved a duality theorem in [9]. M. Schechter [7] has derived a duality theorem in Wolfe's problem without assuming the differentiability of the objective function and the constraint functions. If $A=X$, the authors have proved a duality theorem, assuming that $f$ is strictly convex, between the problems (P) and (D) in [5].

In this paper, we shall derive, in Section 2, a set of necessary and sufficient conditions of Kuhn-Tucker type for a point to be optimal for problem (P). We shall use this generalized Kuhn-Tucker theorem to prove a duality and a converse duality theorem between the problems (P) and (D) in Section 3. In Section 4, we apply these theorems in the case of the objective function is the sum of a continuous convex function and a positively homogeneous, lower-semi-continuous, convex function.

\section{Preliminaries}

In this paper $X$ and $X^{\prime}$, as well as $Z$ and $Z^{\prime}$, shall be pairs of real vector spaces in duality, with their respective weak topologies. Thus all the spaces will be locally convex spaces. We denote by $\langle\cdot, \cdot\rangle$ the canonical bilinear form of the dualities between the spaces $X$ and $X^{\prime}$, as well as $Z$ and $Z^{\prime}$. We let $H \subset Z$ be a closed convex cone with non-empty interior defining a partial order in $Z$-for $x, y \in Z$; $x \leqslant y$ if $y-x \in H$. For $x, y \in Z, x<y$ is equivalent to $y-x$ is an interior point of $H$. Let $H^{*}$ stand for the conjugate cone, namely,

$$
H^{*}=\left\{z^{*} \in Z^{\prime}:\left\langle z^{*}, z\right\rangle \geqslant 0 \text { for every } z \in H\right\} .
$$

Then, $H^{*}$ defines a partial order in $Z^{\prime}$. 
Let $G: X \rightarrow Z$ be an operator. $G$ is said to be convex if

$$
G(t x+(1-t) y) \leqslant t G(x)+(1-t) G(y),
$$

for all $x, y \in X$ and $0 \leqslant t \leqslant 1$.

A continuous linear operator $T: X \rightarrow Z$ is said to be a subgradient of $G$ at a point $x_{0} \in X$ if

$$
T\left(x-x_{0}\right) \leqslant G(x)-G\left(x_{0}\right)
$$

for every $x \in X$. The set of all subgradients of $G$ at $x_{0}$ is called the subdifferential of $G$ at $x_{0}$ and is denoted by $\partial G\left(x_{0}\right)$.

The operator $G: X \rightarrow Z$ is said to be regularly subdifferentiable at $x_{0}$ if

$$
\partial\left(z^{*} \circ G\right)\left(x_{0}\right)=z^{*} \circ \partial G\left(x_{0}\right)
$$

for every $z^{*} \in H^{*}[1]$. If $G$ is regularly subdifferentiable at every point of a subset $A$ of $X$, then $G$ is said to be regularly subdifferentiable on $A$.

We need the following proposition, whose proof can be found in [4].

Proposition 1.1. Let $F$ be a positively homogeneous, lower-semicontinuous, convex function defined on a locally convex space $V$; and let $u \neq 0$. Then

$$
\partial F(u)=\left\{u^{*} \in \partial F(0): F(u)=\cdot\left\langle u, u^{*}\right\rangle\right\} .
$$

We shall also need the following definition and a lemma, which can be proved easily.

Definition. Let $f: X \rightarrow R$ be a function, and let $a \in X$. $f$ is said to be strictly convex at $a$ if

$$
f(t a+(1-t) b)<t f(a)+(1-t) f(b)
$$

for every $a \neq b \in X, 0<t<1$.

LeMma 1.2. Let $f: X \rightarrow R$ be convex. If $f$ is strictly convex at $a \in X$, then for every $u^{*} \in \partial f(a)$, we have

$$
f(x)-f(a)>\left\langle u^{*}, x-a\right\rangle
$$

for every $x \in X, x \neq a$.

\section{Necessary and sufficient conditions}

Before establishing a necessary and sufficient condition of Kuhn-Tucker type, we shall prove a theorem of Fritz-John type. 
THEOREM 2.1. Let $X$ be a locally convex space and let $f$ be a convex function, continuous at a point of the convex set $A$ and let $Z$ be a locally convex space with $a$ positive cone $H$ with non-empty interior. Let $G$ be a continuous convex operator from $X$ to $Z$, which is regularly subdifferentiable on $A$. If $x_{0}$ is an optimal solution of the problem $(\mathrm{P})$, then there exists $\lambda_{0} \geqslant 0, z_{0}^{*} \in H^{*}$, not both zero, such that

$$
0 \in \lambda_{0} \partial f\left(x_{0}\right)+z_{0}^{*} \circ \partial G\left(x_{0}\right)+N\left(x_{0} / A\right)
$$

and $\left\langle z_{0}^{*}, G\left(x_{0}\right)\right\rangle=0$.

Proof. Consider the set $C$ in $Z \times R$ defined as follows:

$C=\left\{(z, a) \in Z \times R\right.$ : there exists $x \in A$ such that $\left.f(x)-f\left(x_{0}\right)<a, G(x) \leqslant z\right\}$

Since $C$ contains $H \times R^{+}$and $H$ has non-empty interior, $C$ has non-empty interior.

The set $C$ is convex, since $f$ and $G$ are convex. Further $(0,0) \notin C$, for if $(0,0) \in C$, then there exists $x \in A$ such that $f(x)-f\left(x_{0}\right)<0$, and $G(x) \leqslant 0$, which is a contradiction to the assumption that $x_{0}$ is an optimal solution of the problem $(\mathrm{P})$. Hence by separation theorem, there exists $(0,0) \neq\left(z_{0}^{*}, \lambda_{0}\right) \in Z^{\prime} \times R$ such that

$$
\left\langle z_{0}^{*}, z\right\rangle+\dot{\lambda}_{0} a \geqslant 0 \text { for every }(z, a) \in C .
$$

In particular, for every $a>0,\left(G\left(x_{0}\right), a\right) \in C$ and hence we have

$$
\left\langle z_{0}^{*}, G\left(x_{0}\right)\right\rangle+\lambda_{0} a \geqslant 0 .
$$

Letting $a \rightarrow 0^{+}$, we obtain

$$
\left\langle z_{0}^{*}, G\left(x_{0}\right)\right\rangle \geqslant 0 .
$$

From (2) and (3), we have, by contradiction,

$$
\lambda_{0} \geqslant 0 \text {. }
$$

Also for every $h \in H,\left(G\left(x_{0}\right)+h, 1\right) \in C$, so that (1) gives

$$
\left\langle z_{0}^{*}, G\left(x_{0}\right)\right\rangle+\lambda_{0}+\left\langle z_{0}^{*}, h\right\rangle \geqslant 0 .
$$

That is, $\left\langle z_{0}^{*}, h\right\rangle \geqslant-\left[\left\langle z_{0}^{*}, G\left(x_{0}\right)\right\rangle+\lambda_{0}\right]$ for every $h \in H$. Again from (3) and (4), we have by contradiction $z_{0}^{*} \in H^{*}$. But since $G\left(x_{0}\right) \in-H$ and $z_{0}^{*} \in H^{*}$, we have

$$
\left\langle z_{0}^{*}, G\left(x_{0}\right)\right\rangle \leqslant 0 \text {. }
$$

Putting (3) and (5) together, we get

$$
\left\langle z_{0}^{*}, G\left(x_{0}\right)\right\rangle=0
$$

as desired. 
Now $\left(G(x), f(x)-f\left(x_{0}\right)+\varepsilon\right) \in C$, for all $\varepsilon>0$ and for all $x \in A$. Then by (1), we have

$$
\left\langle z_{0}^{*}, G(x)\right\rangle+\lambda_{0}\left(f(x)-f\left(x_{0}\right)+\varepsilon\right) \geqslant 0 \quad \text { for all } x \in A .
$$

Combining with (6), we have

$$
\left\langle z_{0}^{*}, G(x)-G\left(x_{0}\right)\right\rangle+\lambda_{0}\left(f(x)-f\left(x_{0}\right)+\varepsilon\right) \geqslant 0 \text { for all } x \in A .
$$

As $\varepsilon \rightarrow 0$, we have

$$
\left\langle z_{0}^{*}, G(x)-G\left(x_{0}\right)\right\rangle+\lambda_{0}\left(f(x)-f\left(x_{0}\right)\right) \geqslant 0 \text { for all } x \in A .
$$

That is

$$
\lambda_{0} f\left(x_{0}\right)+\left\langle z_{0}^{*}, G\left(x_{0}\right)\right\rangle \leqslant \lambda_{0} f(x)+\left\langle z_{0}^{*}, G(x)\right\rangle \text { for all } x \in A .
$$

Hence $x_{0}$ minimizes the function $\lambda_{0} f(x)+\left\langle z_{0}^{*}, G(x)\right\rangle$ on $A$. That is $x_{0}$ is a solution of the problem:

$$
\underset{x \in X}{\operatorname{minimize}} \lambda_{0} f(x)+\left\langle z_{0}^{*}, G(x)\right\rangle+\delta(x / A) .
$$

Therefore, by Proposition 1, page 81 in [3], we have

$$
0 \in \partial\left(\lambda_{0} f\left(x_{0}\right)+\left\langle z_{0}^{*}, G\left(x_{0}\right)\right\rangle+\delta\left(x_{0} / A\right)\right) .
$$

Since, $f$ and $G$ are continuous and $G$ is regularly subdifferentiable on $A$, by the Moreau-Rockafeller theorem [6],

$$
0 \in \lambda_{0} \partial f\left(x_{0}\right)+z_{0}^{*} \circ \partial G\left(x_{0}\right)+N\left(x_{0} / A\right) .
$$

Hence the theorem.

We shall now prove a theorem of Kuhn-Tucker type.

THEOREM 2.2. In addition to the assumptions of Theorem 2.1, if we further assume that Stater's constraint qualification is satisfied (that is, there exists $x^{\prime} \in A$ such that $G\left(x^{\prime}\right)<0$ ), then $\lambda_{0} \neq 0$ and one can set $\lambda_{0}=1$. In this case, the necessary and sufficient condition for $x_{0}$ to be an optimal solution of the problem $(\mathrm{P})$ is that there exists an $z_{0}^{*} \in H^{*}$ such that

$$
0 \in \partial f\left(x_{0}\right)+z_{0}^{*} \circ \partial G\left(x_{0}\right)+N\left(x_{0} / A\right) \text { and }\left\langle z_{0}^{*}, G\left(x_{0}\right)\right\rangle=0 .
$$

Proof. Suppose Slater's constraint qualification is satisfied. Then there exists $x^{\prime} \in A$ such that $G\left(x^{\prime}\right)<0$.

Since all the conditions of Theorem 2.1 are satisfied, we have by (7) in the proof of Theorem 2.1, there exists $\lambda_{0} \geqslant 0, z_{0}^{*} \in H^{*}$, not both zero such that

$$
\lambda_{0} f\left(x_{0}\right)+\left\langle z_{0}^{*}, G\left(x_{0}\right)\right\rangle \leqslant \lambda_{0} f(x)+\left\langle z_{0}^{*}, G(x)\right\rangle
$$

for all $x \in A$ and $\left\langle z_{0}^{*}, G\left(x_{0}\right)\right\rangle=0$. 
If $\lambda_{0}=0$, then $z_{0}^{*} \neq 0, z_{0}^{*} \in H^{*}$ and we have

$$
\lambda_{0} f\left(x^{\prime}\right)+\left\langle z_{0}^{*}, G\left(x^{\prime}\right)\right\rangle=\left\langle z_{0}^{*}, G\left(x^{\prime}\right)\right\rangle\left\langle 0=\lambda_{0} f\left(x_{0}\right)+\left\langle z_{0}^{*}, G\left(x_{0}\right)\right\rangle\right.
$$

and this contradicts (7). Therefore $\lambda_{0} \neq 0$. Hence we can set $\lambda_{0}=1$ and the relations (8) are satisfied.

Conversely, suppose $x_{0} \in A$ such that $G\left(x_{0}\right) \leqslant 0, z_{0}^{*} \in H^{*}$ satisfy relations (8). Now (8) implies by the Moreau-Rockafellar theorem [6]

$$
0 \in \partial\left(f+z_{0}^{*} \circ G+\delta(\cdot / A)\right)\left(x_{0}\right) .
$$

Then by Proposition 1, page 81 in [3], we have $x_{0}$ is an optimal solution of the problem

$$
\underset{x \in X}{\operatorname{minimize}} f(x)+z_{0}^{*} \circ G(x)+\delta(x / A)
$$

This implies

$$
f\left(x_{0}\right)+z_{0}^{*} \circ G\left(x_{0}\right) \leqslant f(x)+z_{0}^{*} \circ G(x)+\delta(x / A)
$$

for every $x \in X$, as $x_{0} \in A$. Hence,

$$
f\left(x_{0}\right)+z_{0}^{*} \circ G\left(x_{0}\right) \leqslant f(x)+z_{0}^{*} \circ G(x)
$$

for every $x \in A$. Then for any $x \in A$ satisfying $G(x) \leqslant 0$, we have

$$
\begin{aligned}
f\left(x_{0}\right) & =f\left(x_{0}\right)+\left\langle z_{0}^{*}, G\left(x_{0}\right)\right\rangle \leqslant f(x)+\left\langle z_{0}^{*}, G(x)\right\rangle, \text { by }(9) \\
& \leqslant f(x) .
\end{aligned}
$$

This means that $x_{0}$ is an optimal solution of problem (P).

REMARK. If $Z=R^{m}$, then Theorems 2.1 and 2.2 reduce to Theorems 1.1 and 1.2 in [8] proved by $\mathbf{M}$. Schechter using the theory of Dubovitski-Milyutin [2]. If $A=X$, then Theorem 2.2 becomes Theorem 2 in [4].

\section{Duality and converse duality theorems}

Using the necessary conditions of the previous section, we prove a duality theorem and a converse duality theorem between the problems (P) and (D). We assume that the Slater's constraint qualification is satisfied.

THEOREM 3.1 (Duality). If $x_{0}$ is an optimal solution of $(\mathrm{P})$, then there exists an $z_{0}^{*}$ such that $\left(x_{0}, z_{0}^{*}\right)$ is optimal for (D). Further, the two problems have the same extremal values.

Proof. Since $x_{0}$ is an optimal solution of (P), Theorem 2.2 guarantees the existence of feasible solutions to problem (D). 
Let $\left(x, z^{*}\right)$ be a feasible solution for problem (D). Then $z^{*} \geqslant 0$ and $0 \in \partial f(x)$ $+z^{*} \circ \partial G(x)+N(x / A)$. This implies that there exist $x^{*} \in \partial f(x), T \in \partial G(x)$ and $y^{*} \in N(x / A)$ such that $0=x^{*}+z^{*} \circ T+y^{*}$. Now,

$$
\begin{aligned}
f\left(x_{0}\right)-\left[f(x)+\left\langle z^{*}, G(x)\right\rangle\right] \\
=\left[f\left(x_{0}\right)-f(x)\right]-\left\langle z^{*}, G(x)\right\rangle \\
\geqslant\left\langle x^{*}, x_{0}-x\right\rangle-\left\langle z^{*}, G(x)\right\rangle, \text { since } x^{*} \in \partial f(x) \\
=-\left\langle z^{*} \circ T+y^{*}, x_{0}-x\right\rangle-\left\langle z^{*}, G(x)\right\rangle \\
=-\left\langle z^{*}, T\left(x_{0}-x\right)\right\rangle-\left\langle y^{*}, x_{0}-x\right\rangle-\left\langle z^{*}, G(x)\right\rangle \\
\geqslant-\left\langle z^{*}, G\left(x_{0}\right)-G(x)\right\rangle-\left\langle y^{*}, x_{0}-x\right\rangle-\left\langle z^{*}, G(x)\right\rangle \\
=-\left\langle z^{*}, G\left(x_{0}\right)\right\rangle-\left\langle y^{*}, x_{0}-x\right\rangle \\
\geqslant 0 \quad\left(\text { since } z^{*} \geqslant 0, G\left(x_{0}\right) \leqslant 0 \text { and } y^{*} \in N(x / A)\right) .
\end{aligned}
$$

Thus,

$$
f\left(x_{0}\right) \geqslant f(x)+\left\langle z^{*}, G(x)\right\rangle
$$

for any feasible solution $\left(x, z^{*}\right)$ for problem (D). Since $x_{0}$ is an optimal solution of (P), we have from Theorem 2, that there exists $z_{0}^{*} \in H^{*}$ such that $\left\langle z_{0}^{*}, G\left(x_{0}\right)\right\rangle$ $=0$ and $0 \in \partial f\left(x_{0}\right)+z_{0}^{*} \circ \partial G\left(x_{0}\right)+N\left(x_{0} / A\right)$. In other words, $\left(x_{0}, z_{0}^{*}\right)$ is a feasible solution for $(D)$. Hence

$$
f\left(x_{0}\right)=f\left(x_{0}\right)+\left\langle z_{0}^{*}, G\left(x_{0}\right)\right\rangle .
$$

Thus, from (1) and (2), $\left(x_{0}, z_{0}^{*}\right)$ is an optimal solution of problem (D), and that the two problems have the same extremal value.

THEOREM 3.2 (Converse Duality). Let us assume that the primal problem (P) has a solution $\bar{x}$. If $\left(x_{0}, z_{0}^{*}\right)$ is an optimal solution of the dual problem (D), and if $f$ is strictly convex at $x_{0}$, then $x_{0}=\bar{x}$. Hence $x_{0}$ solves the problem (P). Furthermore, the extremal values of the two problems are same.

Proof. Suppose $x_{0} \neq \bar{x}$. Since $\bar{x}$ is a solution of (P), it follows from the duality Theorem 3.1, there exists $\bar{z}^{*} \in H^{*}$ such that $\left(\bar{x}, \bar{z}^{*}\right)$ is optimal for (D).

Let $L\left(x, z^{*}\right)=f(x)+\left\langle z^{*}, G(x)\right\rangle$ be the Lagrangian of $(\mathrm{P})$. Then,

$$
L\left(\bar{x}, \vec{z}^{*}\right)=L\left(x_{0}, z_{0}^{*}\right)=\max _{\left(x, z^{*}\right) \in K} L\left(x, z^{*}\right)
$$

where $K=\left\{\left(x, z^{*}\right): x \in A, z^{*} \in H^{*}\right.$ and $\left.0 \in \partial f(x)+z^{*} \circ \partial G(x)+N(x / A)\right\}$. Note that $\left(\bar{x}, \bar{z}^{*}\right) \in K$. 
Since $\left(x_{0}, z_{0}^{*}\right) \in K$, we have $0 \in \partial f\left(x_{0}\right)+z_{0}^{*} \circ \partial G\left(x_{0}\right)+N\left(x_{0} / A\right)$. Hence there exist $x^{*} \in \partial f\left(x_{0}\right), T \in \partial G\left(x_{0}\right)$ and $y^{*} \in N\left(x_{0} / A\right)$ such that $0=x^{*}+$ $z_{0}^{*} \circ T+y^{*}$. Now,

$$
\begin{aligned}
L\left(\bar{x}, z_{0}^{*}\right) & -L\left(x_{0}, z_{0}^{*}\right)=f(\bar{x})+\left\langle z_{0}^{*}, G(\bar{x})\right\rangle-f\left(x_{0}\right)-\left\langle z_{0}^{*}, G\left(x_{0}\right)\right\rangle \\
& =f(\bar{x})-f\left(x_{0}\right)+\left\langle z_{0}^{*},-G(\bar{x})-G\left(x_{0}\right)\right\rangle \\
& >\left\langle x^{*}, \bar{x}-x_{0}\right\rangle+\left\langle z_{0}^{*}, G(\bar{x})-G\left(x_{0}\right)\right\rangle, \quad \text { by Lemma } 1.2, \\
& \geqslant\left\langle x^{*}, \bar{x}-x_{0}\right\rangle+\left\langle z_{0}^{*}, T(\bar{x})-T\left(x_{0}\right)\right\rangle, \quad \text { since } T \in \partial G\left(x_{0}\right) \\
& =\left\langle x^{*}, \bar{x}-x_{0}\right\rangle+\left\langle z_{0}^{*} \circ T, \bar{x}-x_{0}\right\rangle \\
& =+\left\langle x^{*}+z_{0}^{*} \circ T, \bar{x}-x_{0}\right\rangle \\
& =-\left\langle y^{*}, \bar{x}-x_{0}\right\rangle \text { by }(1) \\
& \geqslant 0, \text { since } y^{*} \in N\left(x_{0} / A\right) .
\end{aligned}
$$

It follows that, $L\left(\bar{x}, z_{0}^{*}\right)>L\left(x_{0}, z_{0}^{*}\right)=L\left(\bar{x}, \bar{z}^{*}\right)$. That is,

$$
f(\bar{x})+\left\langle z_{0}^{*}, G(\bar{x})\right\rangle>f(\bar{x})+\left\langle\bar{z}^{*}, G(\bar{x})\right\rangle .
$$

By hypothesis, since $\bar{x}$ is a solution of $(\mathrm{P})$, it follows from Theorem $2,\left\langle\bar{z}^{*}, G(\bar{x})\right\rangle$ $=0$. Hence, by $(3),\left\langle z_{0}^{*}, G(\bar{x})\right\rangle>0$, which is a contradiction to the fact that $z_{0}^{*} \in H^{*}, G(\bar{x}) \leqslant 0$. Hence, $\bar{x}=x_{0}$ and $x_{0}$ solves the problem $(\mathrm{P})$.

Further, we have, $f\left(x_{0}\right)=f(\bar{x})=f(\bar{x})+\left\langle\bar{z}^{*}, G(\bar{x})\right\rangle=L\left(\bar{x}, \bar{z}^{*}\right)=L\left(x_{0}, z_{0}^{*}\right)$ $=f\left(x_{0}\right)+\left\langle z_{0}^{*}, G\left(x_{0}\right)\right\rangle$. Hence, the extremal values of the two problems are equal.

\section{Applications}

We shall now specialize the theorems derived in Section 3 to the case where the objective function is the sum of a positively homogeneous, lower-semi-continuous convex function and a continuous convex function.

Let the objective function $f: X \rightarrow R$ be of the form $f=f_{1}+f_{2}$, where $f_{1}$ is a continuous convex function and $f_{2}$ is a positively homogeneous lower-semicontinuous convex function. Then the problem $(\mathrm{P})$ becomes

$$
\begin{gathered}
\left(\mathrm{P}_{1}\right): \text { Minimize } f_{1}(x)+f_{2}(x) \text { subject to } \\
G(x) \leqslant 0, \text { and } x \in A .
\end{gathered}
$$

Let us now construct the dual problem $\left(D_{1}\right)$ using the above argument.

$$
\begin{gathered}
\left(\mathrm{D}_{1}\right) \text { : Maximize } f_{1}(x)+\left\langle u^{*}, x\right\rangle+\left\langle z^{*}, G(x)\right\rangle \text { subject to } \\
s^{*} \geqslant 0, u^{*} \in \partial f_{2}(0),\left\langle u^{*}, x\right\rangle=f_{2}(x), x \in A \text { and } \\
0 \in \partial f_{1}(x)+u^{*}+z^{*} \circ \partial G(x)+N(x / A) .
\end{gathered}
$$


We will now show that the duality theorem still holds even if one of the constraints is removed from the dual problem $\left(\mathrm{D}_{1}\right)$.

$$
\begin{gathered}
\left(\mathrm{D}_{2}\right): \text { Maximize } f_{1}(x)+\left\langle u^{*}, x\right\rangle+\left\langle z^{*}, G(x)\right\rangle \text { subject to } \\
z^{*} \geqslant u^{*} \in \partial f_{2}(0), x \in A \text { and } \\
0 \in \partial f_{1}(x)+u^{*}+z^{*} \circ \partial G(x)+N(x / A) .
\end{gathered}
$$

THEOREM 4.1. If $x_{0}$ is an optimal solution of $\left(\mathrm{P}_{1}\right)$, then there exist $z_{0}^{*}$, $u_{0}^{*}$ and $w_{0}^{*}$ such that $\left(x_{0}, z_{0}^{*}, u_{0}^{*}, w_{0}^{*}\right)$ is optimal for $\left(\mathrm{D}_{2}\right)$. Further, the two problems have the same extremal values.

Proof. Since $x_{0}$ is optimal for $\left(\mathrm{P}_{1}\right)$, by Theorem 2.2 there exists an $z^{*} \in H^{*}$ such that $\left\langle z^{*}, G\left(x_{0}\right)\right\rangle=0$ and $0 \in \partial\left(f_{1}+f_{2}\right)\left(x_{0}\right)+z^{*} \circ \partial G\left(x_{0}\right)+N\left(x_{0} / A\right)$. But $\partial\left(f_{1}+f_{2}\right)\left(x_{0}\right)=\partial f_{1}\left(x_{0}\right)+\partial f_{2}(x)$ by the Moreau-Rockafellar theorem [6]. Also, $\partial f_{2}\left(x_{0}\right)=\left\{u^{*} \in \partial f_{2}(0): f_{2}\left(x_{0}\right)=\left\langle u^{*}, x_{0}\right\rangle\right\}$, by Proposition 1.1. Therefore,

$$
0 \in \partial f_{1}\left(x_{0}\right)+\left\{u^{*} \in \partial f_{2}(0): f_{2}\left(x_{0}\right)=\left\langle u^{*}, x_{0}\right\rangle\right\}+z^{*} \circ \partial G\left(x_{0}\right)+N\left(x_{0} / A\right) \text {. }
$$

Hence, there is $u^{*} \in \partial f_{2}(0)$ satisfying $f_{2}\left(x_{0}\right)=\left\langle u^{*}, x_{0}\right\rangle$ such that $0 \in \partial f_{1}\left(x_{0}\right)+$ $u^{*}+z^{*} \circ \partial G\left(x_{0}\right)+N\left(x_{0} / A\right)$. Thus feasible solutions to problem $\left(\mathrm{D}_{2}\right)$ exist.

Let $\left(x, z^{*}, u^{*}, w^{*}\right)$ be any feasible solution for $\left(\mathrm{D}_{2}\right)$. Then $z^{*} \in H^{*}, u^{*} \in \partial f_{2}(0)$ and there exist $x^{*} \in \partial f_{1}(x), T \in \partial G(x)$ and $w^{*} \in N(x / A)$ such that

$$
0=x^{*}+u^{*}+z^{*} \circ T+w^{*} .
$$

Now, using the idea of subdifferential calculus, the definition of normal cone and the relation ( 1 ), we can easily prove

$$
f_{1}\left(x_{0}\right)+f_{2}\left(x_{0}\right) \geqslant f_{1}(x)+\left\langle u^{*}, x\right\rangle+\left\langle z^{*}, G(x)\right\rangle
$$

for every feasible solution $\left(x, z^{*}, u^{*}, w^{*}\right)$ of $\left(\mathrm{D}_{2}\right)$. Now, since $x_{0}$ is optimal for $\left(\mathrm{P}_{1}\right)$, then there are $z_{0}^{*} \in H^{*}, u_{0}^{*} \in \partial f_{2}(0)$ satisfying $f_{2}\left(x_{0}\right)=\left\langle u_{0}^{*}, x_{0}\right\rangle$ such that $0 \in \partial f_{1}\left(x_{0}\right)+u_{0}^{*}+z_{0}^{*} \circ \partial G\left(x_{0}\right)+N\left(x_{0} / A\right)$ and such that $\left\langle z_{0}^{*}, G\left(x_{0}\right)\right\rangle=0$. Hence $f_{1}\left(x_{0}\right)+f_{2}\left(x_{0}\right)+\left\langle z_{0}^{*}, G\left(x_{0}\right)\right\rangle \geqslant f_{1}(x)+\left\langle u^{*}, x\right\rangle+\left\langle z^{*}, G(x)\right\rangle$ for every feasible solution $\left(x, z^{*}, u^{*}, w^{*}\right)$ of $\left(\mathrm{D}_{2}\right)$. That is, $\left(u_{0}, z_{0}^{*}, u_{0}^{*}, w_{0}^{*}\right)$ is optimal for $\left(D_{2}\right)$. Further, it is clear that the extremal values of the two problems are the same.

REMARK. The $\left(u_{0}, z_{0}^{*}, u_{0}^{*}, w_{0}^{*}\right)$ which optimizes $\mathrm{D}_{2}$, in fact, also optimizes $\mathrm{D}_{1}$.

THEOREM 4.2. Let $\bar{x}$ be an optimal solution of $\left(\mathrm{P}_{1}\right)$. If $\left(x_{0}, z_{0}^{*}, u_{0}^{*}, w_{0}^{*}\right)$ is optimal for $\left(\mathrm{D}_{1}\right)$ and if $f_{1}$ is strictly convex at $x_{0}$, then $x_{0}=\bar{x}$. Hence $x_{0}$ solves $\left(\mathrm{P}_{1}\right)$. Further, the extremal values of the two problems are equal. 
Proof. Suppose $x_{0} \neq \bar{x}$. Since $\bar{x}$ is a solution of $\left(\mathrm{P}_{1}\right)$, it follows from the duality Theorem 4.1, there exist $\bar{z}^{*} \in H^{*}, \bar{u}^{*} \in \partial f_{2}(0)$ satisfying $f_{2}(\bar{x})=\left\langle\bar{u}^{*}, \bar{x}\right\rangle$ and $\bar{w}^{*} \in N(\bar{x} / A)$ such that $0 \in \partial f_{1}(\bar{x})+\bar{u}^{*}+\bar{z}^{*} \circ \partial G(\bar{x})+\bar{w}^{*}$. That is, $\left(\bar{x}, \bar{z}^{*}, \bar{u}^{*}, \bar{w}^{*}\right)$ is optimal for $\left(\mathrm{D}_{1}\right)$.

Let $\phi\left(x, z^{*}, u^{*}\right)=f_{1}(x)+\left\langle u^{*}, x\right\rangle+\left\langle z^{*}, G(x)\right\rangle$. Hence,

$$
\Phi\left(\bar{x}, \bar{z}^{*}, \bar{u}^{*}\right)=\phi\left(x_{0}, z_{0}^{*}, u_{0}^{*}\right)=\max _{\left(x, z^{*}, u^{*}\right) \in N} \phi\left(x, z^{*}, u^{*}\right)
$$

where $N=\left\{\left(x, z^{*}, u^{*}\right): x \in A, z^{*} \in H^{*}, u^{*} \in \partial f_{2}(0)\right.$ satisfying $f_{2}(x)=\left\langle u^{*}, x\right\rangle$ such that $\left.0 \in \partial f_{1}(x)+u^{*}+z^{*} \circ \partial G(x)+N(x / A)\right\}$. Note that $\left(x_{0}, z_{0}^{*}, u_{0}^{*}\right) \in N$.

Since $\left(x_{0}, z_{0}^{*}, u_{0}^{*}\right) \in N$, we have $0 \in \partial f_{1}\left(x_{0}\right)+u_{0}^{*}+z_{0}^{*} \circ \partial G\left(u_{0}\right)+N\left(x_{0} / A\right)$. Hence, there exist $x^{*} \in \partial f_{1}\left(x_{0}\right), T \in \partial G\left(u_{0}\right)$ and $w^{*} \in N\left(x_{0} / A\right)$ such that

$$
0=x^{*}+u_{0}^{*}+z_{0}^{*} \circ T+w^{*} \text {. }
$$

Using the idea of subdifferential calculus, definition of normal cone and using the Lemma 1.2 and relation (3), we can prove,

$$
\begin{aligned}
\phi\left(\bar{x}, z_{0}^{*}, \bar{u}^{*}\right)-\phi\left(x_{0}, z_{0}^{*}, u_{0}^{*}\right) & >-\left\langle u_{0}^{*}, \bar{x}\right\rangle+\left\langle\bar{u}^{*}, \bar{x}\right\rangle-\left\langle w^{*}, \bar{x}-x_{0}\right\rangle \\
& \geqslant-f_{2}(\bar{x})+f_{2}(\bar{x})-\left\langle w^{*}, \bar{x}-x_{0}\right\rangle,
\end{aligned}
$$

since $\bar{u}^{*} \in \partial f_{2}(0)$ satisfying $f_{2}(\bar{x})=\left\langle\bar{u}^{*}, \bar{x}\right\rangle$ and $u_{0}^{*} \in \partial f_{2}(0)$ which implies that $f_{2}(\bar{x}) \geqslant\left\langle u_{0}^{*}, \bar{x}\right\rangle=-\left\langle w^{*}, \bar{x}-x_{0}\right\rangle \geqslant 0$, since $w^{*} \in N\left(x_{0} / A\right)$. Therefore, $\phi\left(\bar{x}, z_{0}^{*}, \bar{u}^{*}\right)>\phi\left(x_{0}, z_{0}^{*}, u_{0}^{*}\right)=\phi\left(\bar{x}, \bar{z}^{*}, \bar{u}^{*}\right)$. That is,

$$
f_{1}(\bar{x})+\left\langle\bar{u}^{*}, \bar{x}\right\rangle+\left\langle z_{0}^{*}, G(\bar{x})\right\rangle>f_{1}(\bar{x})+\left\langle\bar{u}^{*}, \bar{x}\right\rangle+\left\langle\bar{z}^{*}, G(\bar{x})\right\rangle .
$$

Since $\bar{x}$ is an optimal solution of $\left(\mathrm{P}_{1}\right)$, it follows from Theorem $2.2,\left\langle\bar{z}^{*}, G(\bar{x})\right\rangle=0$. Hence $\left\langle z_{0}^{*}, G(\bar{x})\right\rangle>0$, from (4) which is not possible because $z_{0}^{*} \in H^{*}, G(\bar{x}) \leqslant 0$. Hence $\bar{x}=x_{0}$, and $x_{0}$ solves the problem $\left(\mathrm{P}_{1}\right)$.

Further, we have,

$$
\begin{aligned}
f_{1}\left(x_{0}\right)+f_{2}\left(x_{0}\right) & =f_{1}(\bar{x})+f_{2}(\bar{x}) \\
& =f_{1}(\bar{x})+\left\langle\bar{u}^{*}, \bar{x}\right\rangle+\left\langle\bar{z}^{*}, G(\bar{x})\right\rangle \\
& =\phi\left(\bar{x}, \bar{z}^{*}, \bar{u}^{*}\right)=\phi\left(x_{0}, z_{0}^{*}, u_{0}^{*}\right) \\
& =f_{1}\left(x_{0}\right)+\left\langle u_{0}^{*}, x_{0}\right\rangle+\left\langle z_{0}^{*}, G\left(x_{0}\right)\right\rangle .
\end{aligned}
$$

Hence, the extremal values of the two problems are equal.

Remark. We are not able to prove a converse duality between $\left(\mathrm{P}_{1}\right)$ and $\left(\mathrm{D}_{2}\right)$.

Special cases of problems of type $\left(\mathrm{P}_{1}\right)$ with finite dimensional applications have been discussed in $[7,8,5]$.

The authors wish to thank the referee for his useful comments and helpful suggestions. 


\title{
References
}

[1] V. Barbu and Th. Precupanu, Convexity and optimization in Banach spaces (Sijthoff and Noordhoff, The Netherlands, 1978).

[2] I. V. Girsenov, Lectures on mathematical theory of extremum problems, (Springer-Verlag, New York. 1972).

[3] A. D. Ioffe and V. M. Tihomirov, Theory of extremal problems (Studies in Mathematics and its Applications, 6, North-Holland, Amsterdam, New York, Oxford, 1979).

[4] P. Kanniappan and Sundaram M. A. Sastry, 'A duality theorem for non-differentiable convex programming with operatorial constraints', Bull. Austral. Math. Soc. 22 (1980), 145-152.

[5] P. Kanniappan and Sundaram M. A. Sastry, 'A subgradient converse duality theorem for a convex programming', communicated to J. Math. Anal. Appl.

[6] R. T. Rockafellar, 'Extension of Fenchel's duality theorem for convex functions,' Duke Muth. J. 33 (1966), 81-89.

[7] M. Schechter, 'A subgradient duality theorem', J. Math. Anal. Appl. 61 (1977), 850-855.

[8] M. Schechter, 'More on subgradient duality', J. Math. Anal. Appl. 71 (1979), 251-262.

[9] P. Wolfe, 'A duality theorem for non-linear programming', Quart. Appl. Math. 19 (1961), 239-244.

\author{
School of Mathematics \\ Madurai Kamaraj University \\ Madurai-625 021 \\ Tamil Nadu \\ India
}

